\title{
Characterization of glucose oxidation by gold nanoparticles using nanoceria
}

\author{
Nathan J. Lang, Biwu Liu and Juewen Liu* \\ Department of Chemistry and Waterloo Institute for Nanotechnology, University of Waterloo, \\ Waterloo, Ontario, Canada, N2L 3G1 \\ E-mail: liujw@uwaterloo.ca
}

The final publication is available at Elsevier via http://dx.doi.org/10.1016/j.jcis.2014.04.025." (c) 2014.

This manuscript version is made available under the CC-BY-NC-ND 4.0 license http://creativecommons.org/licenses/by-nc-nd/4.0/ 


\section{Abstract.}

Gold nanoparticles (AuNPs) can oxidize glucose, producing hydrogen peroxide and gluconic acid, which are the same products as those generated by glucose oxidase (GOx). In this regard, AuNPs are a nanozyme. Herein, a new colorimetric method is developed to understand the surface chemistry of gold nanoparticles for this oxidation reaction. The color of nanoceria is changed to yellow by the hydrogen peroxide generated glucose oxidation. Using this assay, we find that adsorption of small molecules such as citrate does not deactivate AuNPs, while adsorption of polymers including serum proteins and high molecular weight polyethylene glycol inhibits glucose oxidation. In addition to glucose, AuNPs can also oxidize galactose. Therefore, this reaction is unlikely to be directly useful for glucose detection for biomedical applications. On the other hand, AuNPs might serve as a general oxidase for a broad range of substrates. The glucose oxidation reaction is slower at lower $\mathrm{pH}$. Since the reaction generates an acid product, glucose oxidation becomes slower as the reaction proceeds. The effects of temperature, AuNP size, and reaction kinetics have been systematically studied. This work provides new insights regarding the surface chemistry of AuNPs as a nanozyme. 


\section{Introduction}

Nanozymes are nanoparticles with catalytic activity [1-3]. In the past decade, gold nanoparticles (AuNPs) [4-7], magnetic iron oxide NPs [8], and cerium oxide NPs (nanoceria) have been reported to mimic various enzymes [9-16]. Oxidation of glucose by AuNPs was first reported by Comotti et al in 2004 [4]. In this reaction, oxygen and glucose are consumed to produce gluconic acid and hydrogen peroxide $\left(\mathrm{H}_{2} \mathrm{O}_{2}\right)$ [4-6, 17, 18], which are the same products as those generated by glucose oxidase (GOx). Therefore, AuNPs are a mimic of GOx. The enzyme properties of these two have been systematically compared and the $k_{\text {cat }}$ and $\mathrm{K}_{\mathrm{m}}$ values are reported to be quite similar [6].

Recently, analytical chemists picked up this reaction for biosensor development and many interesting observations were made. For example, the $\mathrm{H}_{2} \mathrm{O}_{2}$ generated during glucose oxidation was used as a reducing agent to react with $\mathrm{HAuCl}_{4}$ [19]. The newly reduced gold is deposited on the original particle surface to produce enlarged AuNPs. The growth of AuNP size is monitored by the shift of the surface plasmon peak or color change $[5,6]$. With a short single-stranded DNA, the glucose oxidation reaction by AuNPs is impeded, which is attributed to the adsorption of DNA onto the gold surface. In addition, it was noticed that AuNP nanozymes seem to be deactivated in the reaction process (so called self-limiting reaction). In other words, the glucose conversion becomes progressively slower as the reaction proceeds. This gives a relatively small turnover number, which may compromise its application. This self-limiting behavior was attributed to the capping of AuNP surface by the gluconate product [4-6]. Both DNA adsorption and gluconate adsorption indicate the importance of the surface chemistry of AuNPs for catalysis.

Despite these progresses, a lot remains to be learned to fully understand AuNP nanozymes. First, we are intrigued by the surface chemistry aspect of this reaction. In particular, we aim to compare the activity of AuNPs as a function of the binding affinity and size of surface ligands. Second, we study the buffer conditions, from which we suggest an alternative explanation for the self-limiting reaction. 
Finally, protein enzymes have excellent substrate specificity. It is unclear whether AuNPs is specific for glucose; a few other sugar molecules have been tested in this work as well.

\section{Materials and Methods}

Chemicals. AuNPs $(5,10,20,30$, and 50 and $100 \mathrm{~nm})$ were purchased from Ted Pella Inc. AuNPs (5, 13, and $50 \mathrm{~nm}$ ) were prepared by $\mathrm{NaBH}_{4}$ or citrate reduction in our own lab. $\mathrm{HAuCl}_{4}$, nanoceria, glucose, sodium gluconate, galactose, fructose, bovine serum, and hydrogen peroxide were purchased from Sigma-Aldrich. Trisodium citrate was from Mandel Scientific Inc (Guelph, Ontario, Canada). Sucrose and all the PEG samples were from VWR. Milli-Q water was used for preparing all the solutions. The original $20 \%$ stock solution of nanoceria has a particle concentration of $860 \mu \mathrm{M}$. We typically dilute it first 47.3 times and then 32.26 times to reach a final particle concentration of $\sim 564$ $\mathrm{nM}$ in the final assay tube.

Preparing AuNPs. $13 \mathrm{~nm}$ AuNPs were prepared by the standard citrate reduction method (particle concentration $10 \mathrm{nM}$ ) [20]. $5 \mathrm{~nm}$ AuNPs were prepared by mixing $125 \mu \mathrm{M} \mathrm{HAuCl} 4$ and $1 \mathrm{mM}$ $\mathrm{NaHCO}_{3}$ with a freshly prepared $100 \mathrm{mM}$ solution of $\mathrm{NaBH}_{4}$, added in $10 \mu \mathrm{L}$ drops. The initial volume was $40 \mathrm{~mL}$. The solution was stirred and cooled with ice throughout the synthesis. The addition was stopped by adding $\sim 240 \mu \mathrm{L}$ of $\mathrm{NaBH}_{4}$. Note that the color stops changing after adding roughly $40 \mu \mathrm{L}$. The molar concentration of the as-prepared $5 \mathrm{~nm}$ AuNP is $\sim 4.4 \mathrm{nM}$.

UV-vis spectroscopy. In a typical assay, $2.2 \mathrm{nM} 5 \mathrm{~nm}$ AuNPs were mixed with $10 \mathrm{mM}$ phosphate buffer ( $\mathrm{pH} \mathrm{8)}$ and $5 \mathrm{mM}$ glucose. After $45 \mathrm{~min}$, the samples were treated with $10 \mathrm{mM} \mathrm{KCN}$ to dissolve the AuNPs, and then mixed with $564 \mathrm{nM}$ (nanoparticle concentration) $\mathrm{CeO}_{2}$. After $\mathrm{CeO}_{2}$ addition, exposure to light was minimized, as this bleaches the yellow color produced by Ce reacting with $\mathrm{H}_{2} \mathrm{O}_{2}$. The samples were scanned using a UV-vis spectrometer (Agilent 8453A). The extinction ratio of 400 $\mathrm{nm} / 290 \mathrm{~nm}$ was used to quantify the amount of $\mathrm{H}_{2} \mathrm{O}_{2}$ produced by the AuNP and glucose reaction. It is important to note that fresh nanoceria may need to be prepared occasionally as we found that the 
spectrum of the nanoceria by itself changed after a few days. It is also important to keep the $\mathrm{pH}$ buffered at roughly 8 for the AuNPs to work properly. To test the effect of AuNP size, 5, 10, 20, 30, 50, and $100 \mathrm{~nm}$ diameter AuNPs were mixed with $5 \mathrm{mM}$ glucose and $10 \mathrm{mM}$ phosphate buffer (pH 8).

Varying reaction conditions. To study the effect of PEG adsorption, AuNP samples were mixed with $1 \mathrm{mM}$ of either PEG 200, 400, 2k, 20k or 35k before the glucose addition. To test sensor specificity, AuNPs were mixed with $5 \mathrm{mM}$ of either sucrose, fructose, galactose, ethylene glycol, or glycerol before glucose was added. To test the effect of citrate, $3 \mathrm{mM}$ trisodium citrate was mixed with the $\mathrm{NaBH}_{4}$ reduced $5 \mathrm{~nm}$ AuNPs before adding glucose. To test the sensor at different pH levels, a $10 \mathrm{mM}$ citrate buffer of either $\mathrm{pH} 4,6$, or 8 was substituted for the $10 \mathrm{mM}$ phosphate buffer used otherwise. $\mathrm{pH}$ 10 and 12 were achieved by adding $\mathrm{NaOH}$ to $\mathrm{pH} 8$ phosphate buffer. To observe the effect of temperature, samples with $5 \mathrm{mM}$ glucose and $5 \mathrm{~nm}$ AuNPs with phosphate buffer were incubated for $45 \mathrm{~min}$ at the appropriate temperature. For these experiments, the sample lids were shut to avoid evaporation of $\mathrm{H}_{2} \mathrm{O}_{2}$ or water.

Monitoring pH change. A $5 \mathrm{~mL}$ sample of $5 \mathrm{~nm}$ AuNPs were prepared without buffer. $\mathrm{pH}$ was monitored using a $\mathrm{pH}$ meter (UltraBasic, Denver Instrument) for $4 \mathrm{~h}$, mixing with a final concentration of $1.5 \mathrm{mM} \mathrm{NaOH}$ approximately at the end of each hour. 


\section{Results and Discussion}

Visual detection. One method to monitor the glucose oxidation reaction is to add $\mathrm{HAuCl}_{4}$ to produce new gold surfaces $[6,21]$. However, this will change the original gold surface chemistry, introducing artifacts for our mechanistic studies. To solve this problem, we developed a new method using nanoceria. Nanoceria normally has a light yellow color at high concentration. At low concentration, it is almost colorless. In the presence of $\mathrm{H}_{2} \mathrm{O}_{2}$, an intense yellow/orange color is generated [22]. The detailed reaction mechanism between nanoceria and $\mathrm{H}_{2} \mathrm{O}_{2}$ is quite complex, since $\mathrm{H}_{2} \mathrm{O}_{2}$ can act as an oxidizing agent, a reducing agent and a ligand [23]. This color change and the related redox reactions have been applied to design various sensors [9, 22, 24-26].

Our reaction scheme is presented in Figure 1A. AuNPs convert glucose into gluconic acid, which dissociates into gluconate and proton. Hydrogen peroxide is also produced in the same process. We employ nanoceria as the colorimetric reporter for $\mathrm{H}_{2} \mathrm{O}_{2}$. Our AuNPs were prepared by reducing $\mathrm{HAuCl}_{4}$ with $\mathrm{NaBH}_{4}$, yielding an average particle diameter of $\sim 5 \mathrm{~nm}$ as characterized by dynamic light scattering (DLS, Figure 1B, red trace). Transmission electron microscopy (TEM) shows that these AuNPs are spherical (Figure S1, Electronic Supplementary Information, ESI). Our nanoceria has a diameter of $\sim 5 \mathrm{~nm}$ as characterized by TEM (Figure 1D), and DLS showed a similar average size of but a broader size distribution (Figure 1B, blue trace). With a concentration of $0.02 \%$ nanoceria (860 nM particle concentration), almost no color is initially perceived by the naked human eye; however adding $\mathrm{H}_{2} \mathrm{O}_{2}$ produces a bright yellow color (Figure $1 \mathrm{~A}$ ). The UV-vis spectrum of fresh nanoceria has a peak at $290 \mathrm{~nm}$. In the presence of $\mathrm{H}_{2} \mathrm{O}_{2}$, this peak disappeared while the absorption at $\sim 400 \mathrm{~nm}$ increases. By subtracting the original nanoceria spectrum (black trace, Figure 1A) from the one after adding $\mathrm{H}_{2} \mathrm{O}_{2}$ (normalizing at $290 \mathrm{~nm}$ ), a difference spectrum with a peak at $400 \mathrm{~nm}$ was obtained (red dashed spectrum). This new species explains the yellow color. We estimated the extinction coefficient of $5 \mathrm{~nm}$ nanoceria (after $\mathrm{H}_{2} \mathrm{O}_{2}$ treatment) to be $10 \times 10^{6} \mathrm{M}^{-1} \mathrm{~cm}^{-1}$ at $400 \mathrm{~nm}$, which is comparable with 
AuNPs of similar size (e.g. $9 \times 10^{6} \mathrm{M}^{-1} \mathrm{~cm}^{-1}$ for $4.6 \mathrm{~nm}$ AuNPs) [27]. Such a high extinction coefficient makes it possible to achieve sensitive visual detection. For subsequent quantitative studies, the ratio of absorbance at $400 \mathrm{~nm}$ over $290 \mathrm{~nm}$ was used to quantify the color change and thus of the amount of hydrogen peroxide produced in a sample. Such ratiometric methods are convenient for quantification and have been used to quantify the color change of AuNPs [28]. Using nanoceria to detect $\mathrm{H}_{2} \mathrm{O}_{2}$ is highly sensitive [22], and in our system we can easily detect 3 parts-per-million $\mathrm{H}_{2} \mathrm{O}_{2}$ (Figure S2).
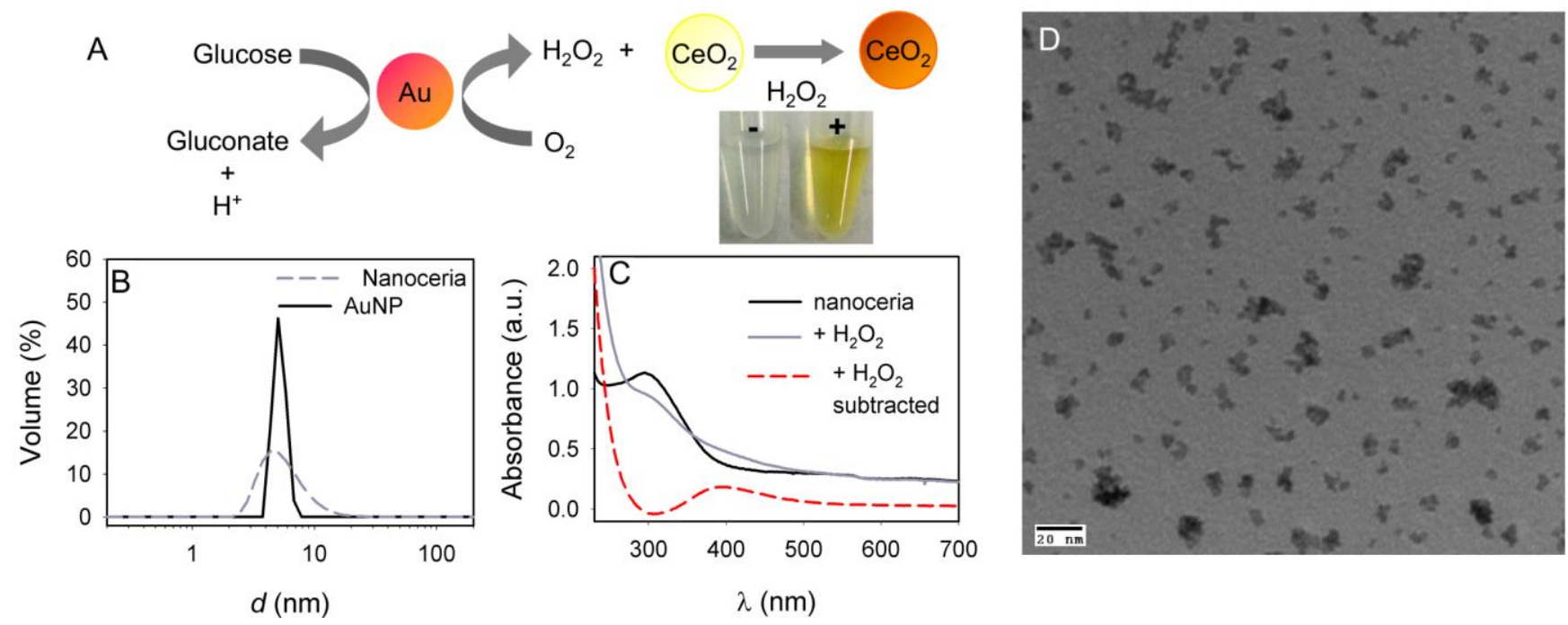

Figure 1. (A) Schematic presentation of AuNP reacting with glucose and oxygen to produce gluconic acid and $\mathrm{H}_{2} \mathrm{O}_{2}$. The $\mathrm{pH}$ decreases during the reaction. A photograph of visible colour change after $\mathrm{H}_{2} \mathrm{O}_{2}$ addition to nanoceria is also shown. (B) DLS spectra of $5 \mathrm{~nm}$ AuNPs and nanoceria used in this work. (C) UV-vis spectra of nanoceria with and without $\mathrm{H}_{2} \mathrm{O}_{2}$ addition. The red dashed spectrum is obtained by subtracting the black spectrum from the red one (normalized at $290 \mathrm{~nm}$ ). (D) TEM image of nanoceria.

Effect of buffer conditions. To under the kinetics of the reaction described in Figure 1A, we mixed glucose with AuNPs and then added nanoceria at designated time points. To minimize the background 
color interference from AuNPs, $\mathrm{KCN}$ was added to dissolve the AuNPs before adding nanoceria. Control experiments showed that $\mathrm{KCN}$ does not interfere with the reaction between nanoceria and $\mathrm{H}_{2} \mathrm{O}_{2}$ (Figure S3). Without $\mathrm{KCN}$, the surface plasmon peak of AuNPs can be observed at $520 \mathrm{~nm}$ (Figure S4). If As expected, higher ratio of absorption at $400 \mathrm{~nm}$ over $290 \mathrm{~nm}$ is observed by using longer incubation time and the reaction reaches a plateau in $30 \mathrm{~min}$ (Figure 2A), where the absorbance ratio is $\sim 0.08$. There is still a lot of room for nanoceria to further change its color, since the ratio could reach $\sim 0.4$ based on the UV-vis spectra in Figure 1C. Since the amount of glucose should be in excess, we reason that the reaction rate is decreased as the reaction proceeds. This reaction kinetics is comparable with that monitored by the increase of AuNP size using $\mathrm{HAuCl}_{4}$ [6]. The similar oxidation rates suggest that both sensors are governed by the same reaction mechanism, where the signal generation step is not the rate limiting step. Increasing temperature decreased the amount of color change in our system (Figure 2B). Glucose oxidation by AuNPs was reported to be faster at higher temperature [18]. Using $\mathrm{HAuCl}_{4}$ for signaling, higher temperature indeed produced faster AuNP growth [6]. Since we added nanoceria after reacting AuNPs with glucose, it is likely that $\mathrm{H}_{2} \mathrm{O}_{2}$ might escape from water at higher temperature. Overall, our reaction was quite stable from room temperature to $\sim 50{ }^{\circ} \mathrm{C}$, where the signal decreased by just $\sim 10 \%$. More drastic change was observed at even higher temperatures. Since the reaction product contains an acid, the $\mathrm{pH}$ may change during the reaction process. Next we studied the effect of $\mathrm{pH}$, where the glucose oxidation reaction was significantly less efficient at lower $\mathrm{pH}$ (Figure $2 \mathrm{C}$ ). The optimal $\mathrm{pH}$ was $\sim 8$, and the rate did not increase further at $\mathrm{pH}$ 10 or 12 . We noted that the AuNPs aggregated at $\mathrm{pH} 12$ since the color turned purple. 

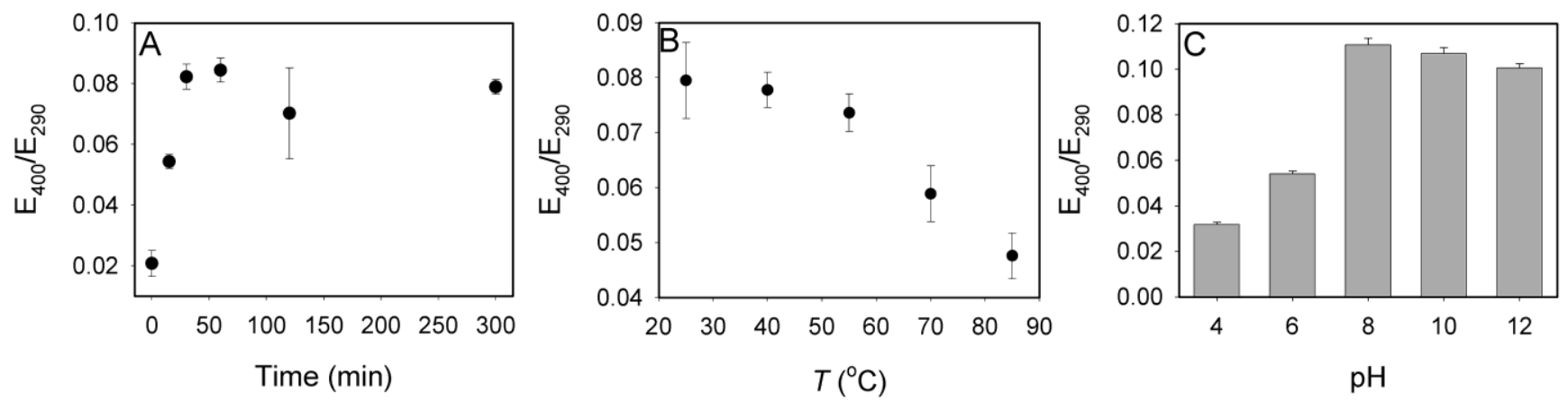

Figure 2. Optimization of glucose detection conditions. (A) Glucose conversion after various time intervals. Samples contained $10 \mathrm{mM}$ glucose, $6 \mathrm{nM} \mathrm{CeO}_{2}$ and $1.25 \mathrm{nM}$ of $5 \mathrm{~nm}$ AuNPs. (B) Effect of temperature on glucose conversion. (C) Effect of $\mathrm{pH}$ on glucose conversion.

pH-limited reaction. Since nanoceria provides a convenient assay to study glucose oxidation by AuNPs, we further used this reaction to understand the surface chemistry of AuNPs during the reaction process. Previous reports showed that the reaction is self-limited. For example, the enlargement of AuNPs in the presence of $\mathrm{HAuCl}_{4}$ became gradually inhibited as the reaction proceeded [6]. This selflimiting behavior was also observed in our nanoceria signaling method, since the absorbance ratio barely reached 0.1 , while this ratio could reach 0.5 with sufficient amount of $\mathrm{H}_{2} \mathrm{O}_{2}$. Therefore, a challenge in improving this sensor is to increase catalytic turnover.

Figure $2 \mathrm{~A}$ shows that the reaction is basically stopped at $30 \mathrm{~min}$. It is unlikely that all the glucose has been consumed at this point since even higher glucose concentration produced the same response (see below). Therefore, product inhibition should be the reason for the lack of more glucose conversion after $30 \mathrm{~min}$. Since gluconic acid is the only other product in addition to $\mathrm{H}_{2} \mathrm{O}_{2}$, it was proposed that gluconate was adsorbed by AuNPs to inhibit the reaction, which has been confirmed by XPS spectroscopy [6]. This conclusion was made based on the AuNP enlargement reaction, which involves the deposition of new gold and is quite different from the direct measurement of $\mathrm{H}_{2} \mathrm{O}_{2}$ in our system. To test this in our system, we directly added sodium gluconate to the AuNPs before adding 
glucose. Interestingly, the inhibition effect of gluconate was minimal (Figure 3A), since all the samples with up to $5 \mathrm{mM}$ gluconate showed similar ratios. Figure $3 \mathrm{C}$ shows the color of the samples with and without $5 \mathrm{mM}$ gluconate. Therefore, the inhibition is unlikely to be related to the direct adsorption of gluconate in our system. Since gluconic acid also produces protons and we know that the reaction is significantly slower at lower $\mathrm{pH}$ (Figure 2C), we further tested $\mathrm{pH}$ change. In a tube containing a $5 \mathrm{~mL}$ sample, we started with a $\mathrm{pH}$ value of 8.05 which was the initial $\mathrm{pH}$ of the $5 \mathrm{~nm}$ gold. Within $10 \mathrm{~min}$ of adding $25 \mathrm{mM}$ glucose, the $\mathrm{pH}$ dropped to $\sim 7.0$ and further incubation resulted in the $\mathrm{pH}$ dropping to 6.7 in $1 \mathrm{~h}$ (Figure 3B). The $\mathrm{pH}$ was easily brought back up by adding $\mathrm{NaOH}$ and $\mathrm{pH}$ drop was again observed. This process can be cycled many times. Each time, a final concentration of $1.5 \mathrm{mM} \mathrm{NaOH}$ was added, suggesting that $1.5 \mathrm{mM}$ glucose was converted. Since our AuNP concentration was $\sim 0.1$ $\mu \mathrm{M}$, each AuNP was able to turnover approximately 1,500 glucose molecules in $1 \mathrm{~h}$. To explain the kinetics in Figure 2A, it is likely that as the rate of $\mathrm{H}_{2} \mathrm{O}_{2}$ production is reduced as the $\mathrm{pH}$ drops, there is more time for the $\mathrm{H}_{2} \mathrm{O}_{2}$ to evaporate. Therefore, despite there being a continuous increase in the quantity of $\mathrm{H}_{2} \mathrm{O}_{2}$ in the system, waiting for longer time does not help to improve the signal. We need to point out that while $\mathrm{pH}$ plays a major role in our system with nanoceria-based detection, the effect of gluconate is likely to be more important in other systems such as the catalyzed growth of AuNPs, where $\mathrm{pH}$ was found to have minimal effects on the AuNP enlargement reaction [6].

Glucose sensing. With these understandings, we next tested the use of this system to detect glucose. Since both the sensing component (AuNPs) and the signaling component (nanoceria) are nanoparticles, this system could be a robust sensor without any biomolecules. As shown in Figure 3D, we observed a yellow color with just $1 \mathrm{mM}$ glucose in 30 min detection time and saturated color was observed with glucose concentration higher than $5 \mathrm{mM}$. This was again attributed to $\mathrm{pH}$ change, retarding further reactions. The quantification is shown in Figure $3 \mathrm{E}$ and the detection limit is determined to be $0.3 \mathrm{mM}$ glucose. To confirm specificity, we mixed the AuNPs with sucrose, fructose, galactose, glycerol or 
ethylene glycol, where strong signal was observed only with galactose besides glucose (Figure 3F). Galactose is the C-4 epimer of glucose and AuNPs do not have the ability to distinguish between these two. In this regard, GOx has better selectivity since galactose is not a good substrate for it [29]. Therefore, while AuNPs mimics GOx, they cannot be used as a sensor for glucose at its current form due to the lack of specificity. On the other hand, it might be a general oxidase with a broad substrate range.

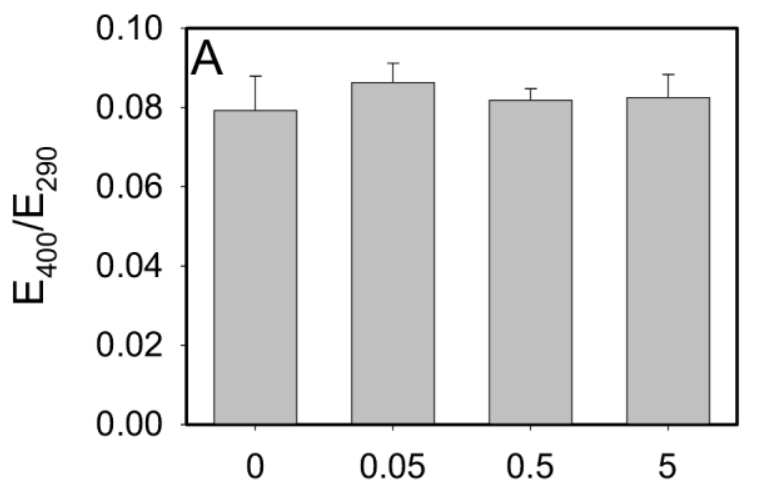

[Gluconate] (mM)
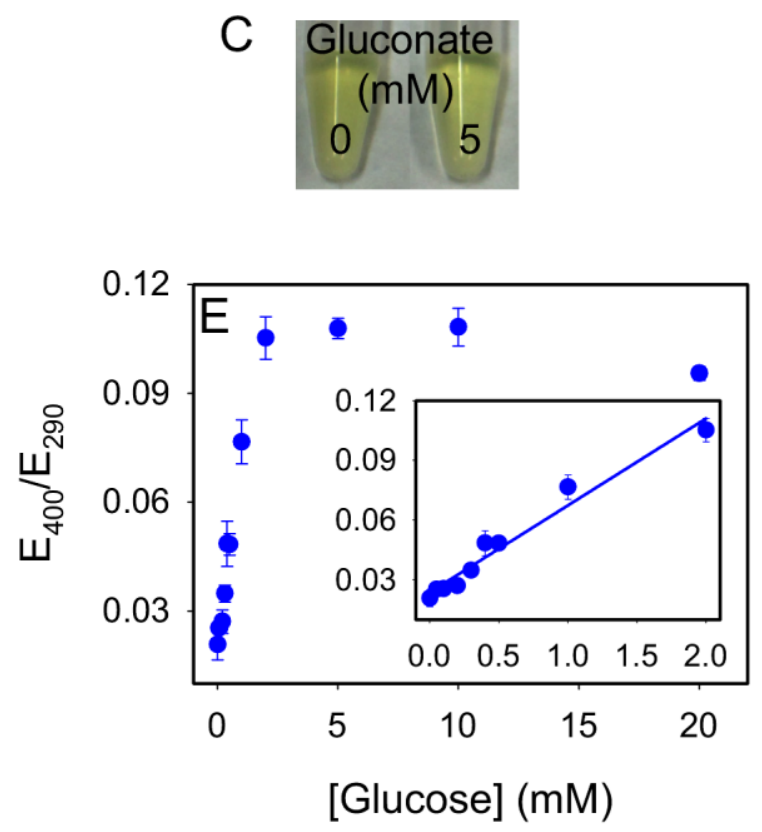
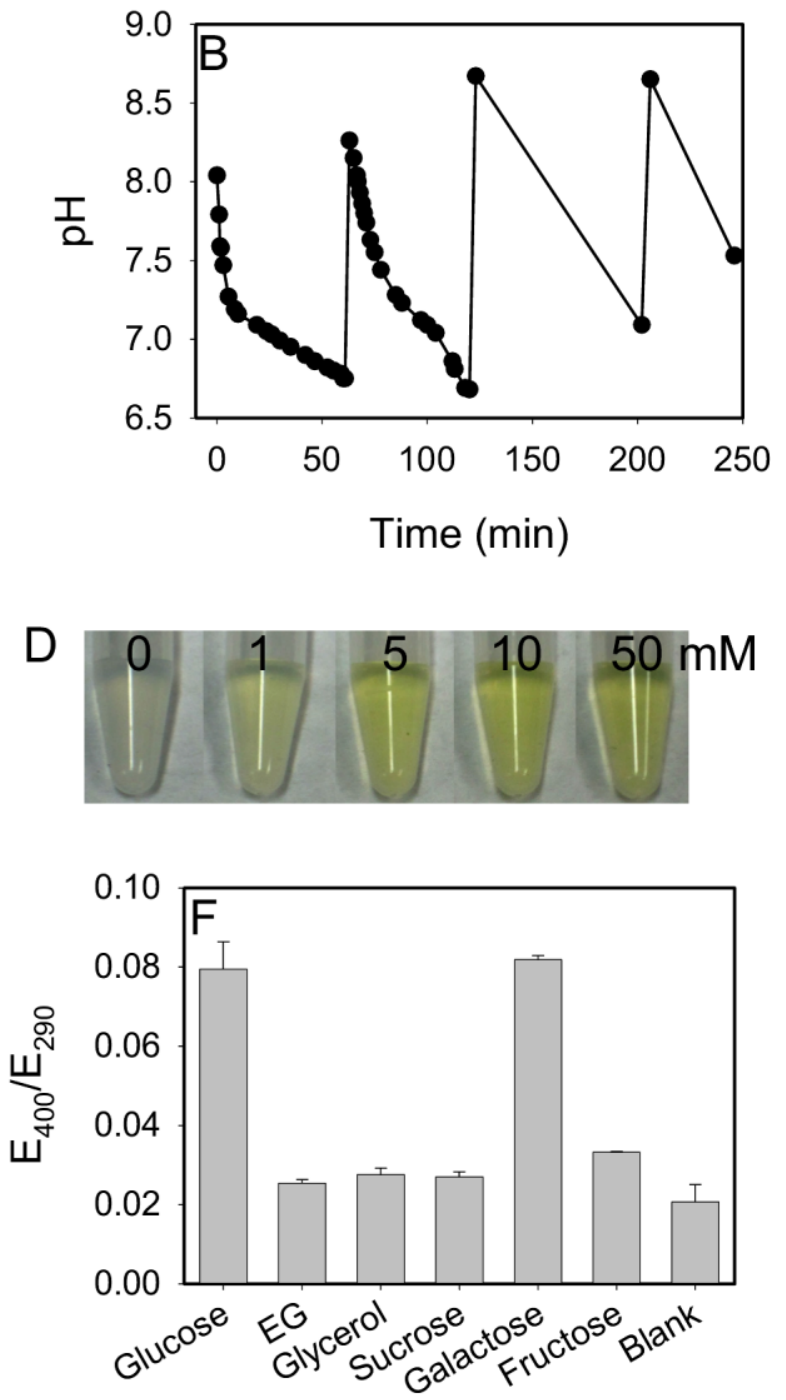
Figure 3. (A) Added sodium gluconate has little inhibition effect on the glucose oxidation reaction. (B) Change of $\mathrm{pH}$ as a function of time during glucose oxidation by AuNPs. $\mathrm{NaOH}(1.5 \mathrm{mM})$ was added at 60, 123, and $206 \mathrm{~min}$. (C) Color of nanoceria without or with $5 \mathrm{mM}$ gluconate for samples in (A). The photograph (D) and absorbance ratio (E) of nanoceria in the presence of various concentration of glucose. (F) The absorbance ratio of nanoceria in various compounds to test selectivity. For the absorbance ratio measurement, each samples contained $564 \mathrm{nM}$ nanoceria, while for imaging, each samples contained $6.06 \mu \mathrm{M} \mathrm{CeO}_{2}$.

Understanding nanoparticle surface. Our above studies have revealed an important reason for reaction inhibition, which is related to $\mathrm{pH}$ change. Next we aim to further understand the surface chemistry of AuNPs in the reaction process. First, we studied the effect of AuNP size and its surface ligand. For a fair comparison, we chose to use the same gold atom concentration, where smaller AuNPs are to have a higher molar concentration and surface area. We prepared $5 \mathrm{~nm}$ AuNPs by $\mathrm{NaBH}_{4}$ reduction, and 13 and $50 \mathrm{~nm}$ AuNPs using citrate. Commercial AuNPs from 5 to $100 \mathrm{~nm}$ were also tested. As shown in Figure 4A, our $5 \mathrm{~nm}$ AuNPs have the highest activity, followed by the commercial $5 \mathrm{~nm}$ AuNPs. Overall, larger AuNPs have lower activity since they have much smaller surface area. Since it is impossible to directly prepare $5 \mathrm{~nm}$ AuNPs using citrate reduction, to test the effect of citrate, we added citrate to our $\mathrm{NaBH}_{4}$ reduced AuNPs and similar activity was obtained (Figure 4B). Therefore, citrate adsorption does not inhibit the reaction. Previous research has clearly indicated that DNA adsorption can inhibit the activity of the AuNPs [5]. We also found that the glucose oxidation reaction was inhibited in just $1 \%$ serum (data not shown), which is attributable to serum protein adsorption. It is likely that blocking AuNP surface by macromolecules can inhibit the activity. This observation also excluded using AuNPs directly for detecting glucose in biological samples. To further understand it, we mixed AuNPs with polyethylene glycol (PEG), which is known to only weakly bind 
to AuNPs [30]. As shown in Figure 4C, PEG inhibits the reaction in a molecular weight dependent manner, where significant inhibition was observed at MW higher than 2000. Previous reaction mechanistic studies have suggested that the glucose oxidation reaction follows the Eley-Rideal mechanism and adsorbed glucose reacts with oxygen in solution [18]. Therefore, polymers binding to AuNPs may inhibit the accessibility of glucose. On the other hand, citrate is a much weaker small molecule ligand that can be displaced by glucose. The fact that low MW PEGs (e.g. PEG 200 and 400) do not inhibit the reaction supports this hypothesis.
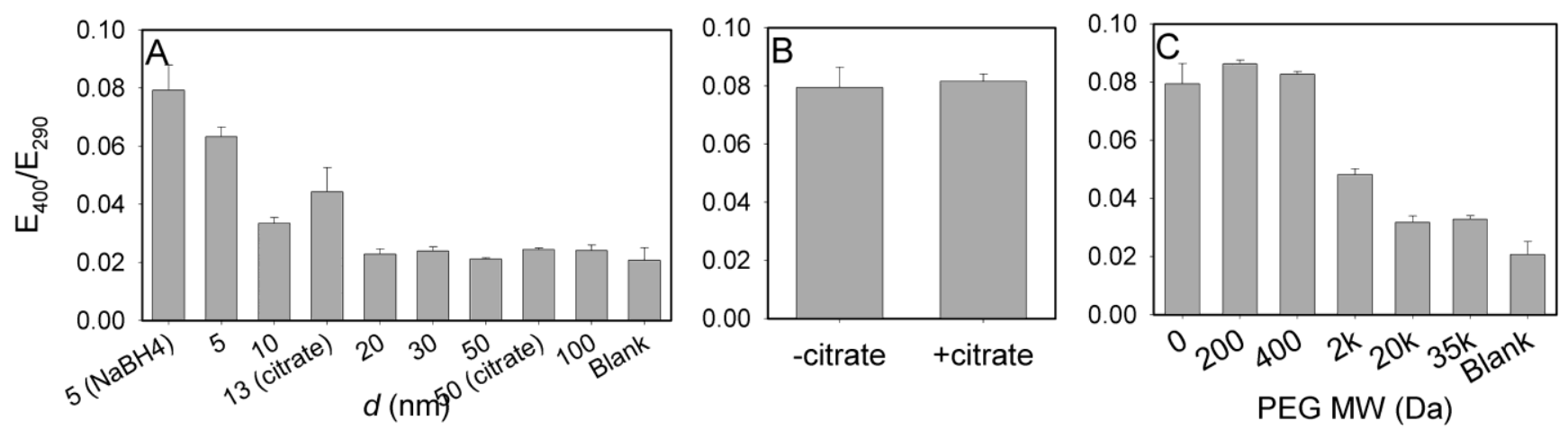

Figure 4. (A) Effect of AuNP size and preparation chemistry on the oxidation of glucose. The first bar is the $5 \mathrm{~nm}$ AuNPs prepared by reducing with $\mathrm{NaBH}_{4}$, the fourth bar is the $13 \mathrm{~nm}$ AuNPs prepared by citrate reduction and the eighth bar is $50 \mathrm{~nm}$ AuNP also from citrate reduction. All the other samples were from commercial sources. Blank means no AuNP. All the samples contained roughly the same gold atom concentration. (B) Comparison of $5 \mathrm{~nm} \mathrm{NaBH}_{4}$ reduced AuNPs in the absence or presence of $3 \mathrm{mM}$ sodium citrate, indicating that citrate adsorption does not inhibit glucose oxidation. (C) Effect of PEG on the oxidation of glucose by $5 \mathrm{~nm}$ AuNPs. 


\section{Conclusions}

In summary, we have revealed a number of important properties of AuNPs relating to their use as a GOx mimic. First, the reaction is slower at lower $\mathrm{pH}$ and glucose oxidation produces acidic products, thus forming a self-limiting system. The reaction can proceed for thousands of turnovers on each AuNP and the $\mathrm{pH}$ drop can be compensated by adding base. Second, by coupling the reaction of nanoceria with hydrogen peroxide and the glucose oxidation by AuNPs, we presented an all-nanoparticle-based assay for studying this reaction. We demonstrated that AuNPs can oxide not only glucose but also galactose, thus serving as a general oxidase. For this reason and for the inhibited activity by polymer adsorption, AuNPs are unlikely to be used for glucose detection. Third, we studied the surface chemistry of AuNPs and found that small molecule ligands containing multiple hydroxide or oxygen groups do not bind to the AuNPs surface strong enough to inhibit the reaction. Even with a relatively common ligand citrate, the AuNPs can still catalyze the reaction at the same rate. On the other hand, even weakly adsorbed polymers such as PEG can effectively inhibit glucose oxidation.

Supplementary Information: TEM of AuNPs and nanoceria reacting with $\mathrm{H}_{2} \mathrm{O}_{2}$.

Acknowledgements. Funding for this work is from the University of Waterloo, the Canadian Foundation for Innovation, and Natural Sciences and Engineering Research Council of Canada (NSERC). J. Liu receives Early Researcher Award from the Ontario Ministry of Research and Innovation. 


\section{References}

1. $\quad$ N. A. Kotov, Science 330 (2010) 188.

2. H. Wei; E. Wang, Chem. Soc. Rev. 42 (2013) 6060.

3. C. J. Brown; R. G. Bergman; K. N. Raymond, J. Am. Chem. Soc. 131 (2009) 17530.

4. $\quad$ M. Comotti; C. Della Pina; R. Matarrese; M. Rossi, Angew. Chem., Int. Ed. 43 (2004) 5812.

5. X. Zheng; Q. Liu; C. Jing; Y. Li; D. Li; W. Luo; Y. Wen; Y. He; Q. Huang; Y.-T. Long; C. Fan, Angew. Chem., Int. Ed. 50 (2011) 11994.

6. W. Luo; C. Zhu; S. Su; D. Li; Y. He; Q. Huang; C. Fan, ACS Nano 4 (2010) 7451.

7. X. Li; Z. Qi; K. Liang; X. Bai; J. Xu; J. Liu; J. Shen, Catal. Lett. 124 (2008) 413.

8. $\quad$ X. Li; F. Wen; B. Creran; Y. Jeong; X. Zhang; V. M. Rotello, Small 8 (2012) 3589.

9. A. Asati; S. Santra; C. Kaittanis; S. Nath; J. M. Perez, Angew. Chem., Int. Ed. 48 (2009) 2308.

10. J. Chen; S. Patil; S. Seal; J. F. McGinnis, Nat Nano 1 (2006) 142.

11. T. Pirmohamed; J. M. Dowding; S. Singh; B. Wasserman; E. Heckert; A. S. Karakoti; J. E. S. King; S. Seal; W. T. Self, Chem. Comm. 46 (2010) 2736.

12. C. Korsvik; S. Patil; S. Seal; W. T. Self, Chem. Comm. (2007) 1056.

13. Y. Peng; X. Chen; G. Yi; Z. Gao, Chem. Comm. 47 (2011) 2916.

14. R. Pautler; E. Y. Kelly; P.-J. J. Huang; J. Cao; B. Liu; J. Liu, ACS Appl. Mater. Inter. 5 (2013) 6820.

15. C. Xu; Z. Liu; L. Wu; J. Ren; X. Qu, Adv. Funct. Mater. 24 (2014) 1624.

16. C. Xu; X. Qu, NPG Asia Mater. 6 (2014) e90.

17. D. Zeng; W. Luo; J. Li; H. Liu; H. Ma; Q. Huang; C. Fan, Analyst 137 (2012) 4435.

18. P. Beltrame; M. Comotti; C. Della Pina; M. Rossi, Applied Catalysis A: General 297 (2006) 1.

19. R. de la Rica; M. M. Stevens, Nat Nano 7 (2012) 821.

20. J. Liu; Y. Lu, Nat. Protoc. 1 (2006) 246.

21. Q. Jiang; Z.-G. Wang; B. Ding, Small 9 (2013) 1016. 
22. M. Ornatska; E. Sharpe; D. Andreescu; S. Andreescu, Anal. Chem. 83 (2011) 4273.

23. P. Yu; S. A. Hayes; T. J. O'Keefe; M. J. O'Keefe; J. O. Stoffer, J. Electrochem. Soc. 153 (2006) C74.

24. E. Sharpe; T. Frasco; D. Andreescu; S. Andreescu, Analyst 138 (2013) 249.

25. J. Njagi; C. Ispas; S. Andreescu, Anal. Chem. 80 (2008) 7266.

26. J. Njagi; M. M. Chernov; J. C. Leiter; S. Andreescu, Anal. Chem. 82 (2010) 989.

27. X. O. Liu; M. Atwater; J. H. Wang; Q. Huo, Colloid. Surface. B. 58 (2007) 3.

28. J. W. Liu; Y. Lu, J. Am. Chem. Soc. 125 (2003) 6642.

29. R. Bentley, Nature 176 (1955) 870.

30. X. Zhang; P.-J. J. Huang; M. R. Servos; J. Liu, Langmuir 28 (2012) 14330. 